\title{
Existence of Solutions for a Class of Quasilinear Parabolic Equations with Superlinear Nonlinearities
}

\author{
Zhong-Xiang Wang, Gao Jia, and Xiao-Juan Zhang \\ College of Science, University of Shanghai for Science and Technology, Shanghai 200093, China \\ Correspondence should be addressed to Gao Jia; gaojia89@163.com
}

Received 13 August 2014; Accepted 23 November 2014; Published 21 December 2014

Academic Editor: Yuncheng You

Copyright (C) 2014 Zhong-Xiang Wang et al. This is an open access article distributed under the Creative Commons Attribution License, which permits unrestricted use, distribution, and reproduction in any medium, provided the original work is properly cited.

Working in a weighted Sobolev space, this paper is devoted to the study of the boundary value problem for the quasilinear parabolic equations with superlinear growth conditions in a domain of $R^{N}$. Some conditions which guarantee the solvability of the problem are given.

\section{Introduction}

In this paper, we deal with the existence of solutions for the quasilinear parabolic problem:

$$
\begin{array}{r}
\rho D_{t} u+\widetilde{\mathscr{M}} u=\lambda_{1} u \rho+f(x, u) \rho-G, \\
(x, t) \in \widetilde{\Omega}, \quad u \in \widetilde{H}(\widetilde{\Omega}, \Gamma),
\end{array}
$$

where $\widetilde{\Omega}=\Omega \times T, \Omega$ is an open set in $R^{N}(N \geq 1), T=(-\pi, \pi)$, $\widetilde{H}(\widetilde{\Omega}, \Gamma)$ is a weighted Sobolev space, $\lambda_{1}$ is the first eigenvalue of $\mathscr{L}$, and $\widetilde{\mathscr{M}}$ is a singular quasilinear operator defined by

$$
\begin{aligned}
\widetilde{M} u= & -\sum_{i, j=1}^{N} D_{i}\left(p_{i}^{1 / 2}(x) p_{j}^{1 / 2}(x) \sigma_{i}^{1 / 2}(u) \sigma_{j}^{1 / 2}(u) b_{i j}(x) D_{j} u\right) \\
& +b_{0}(x) \sigma_{0}(u) q u,
\end{aligned}
$$

where $u(x, t): \widetilde{\Omega} \rightarrow R$. The nonlinear part $f(x, s)$ satisfies the superlinear growth condition

$$
|f(x, s)| \leq h_{0}(x)+K|s|^{\theta-1}, \quad \forall s \in R, \text { a.e. } x \in \Omega,
$$

where $h_{0}(x) \in L_{\rho}^{\theta^{*}}, K$ is a nonnegative constant, $2<\theta<$ $2 N /(N-1)$, and $\theta^{*}=\theta /(\theta-1)$.

There are a number of results concerning solvability of different boundary problems for quasilinear equations (elliptic and parabolic) in which the nonlinearities satisfy sublinear or linear conditions in the weighted Sobolev space, for example, [1-6].

In [1], Shapiro established a new weighted compact Sobolev embedding theorem and proved a series of existence problems for weighted quasilinear elliptic equations and parabolic equations.

In [2], working in Sobolev space $H_{p, \rho}^{1}(\Omega, \Gamma)$ only for the first eigenvalue, Rumbos and Shapiro on the basis of [3] by using the generalized Landesman-Lazer conditions discussed the existence of the solutions for weighted quasilinear elliptic equations

$$
\begin{gathered}
\mathscr{P} u-\lambda_{1} u \rho=-a(x, u) u^{-} \rho+g(x, u) \rho+h, \quad x \text { in } \Omega, \\
u=0, \quad x \text { on } \Omega,
\end{gathered}
$$

where

$$
\mathscr{P} u=-\sum_{i, j=1}^{N} D_{i}\left(p_{i}^{1 / 2} p_{j}^{1 / 2} b_{i j}(x) D_{j} u\right)+\rho(x) c(x) u .
$$

In [4], Jia and Zhao obtained the existence of a nontrivial solution for a class of singular quasilinear elliptic equations in weighted Sobolev spaces.

However, past research results regarding this kind of parabolic equations on superlinearity in the weighted Sobolev space like (1) are very limited. Two notable exceptions are 
found in $[7,8]$, where they discuss the periodic solutions for quasilinear parabolic equations when the nonlinearity may grow superlinearly.

Our goal here is to extend these results to the case of quasilinear parabolic operators.

In fact, (1) is one of the most useful sets which describe the motion of viscous fluid substances. They are widely used in the design of aircrafts and cars, the study of blood flow, the design of power stations, and so forth. Furthermore, coupled with Maxwell's equations, the Navier-Stokes equations can also be used to model and study magnetohydrodynamics.

The main tools applied in our approaches consist of Galerkin method, Brouwer's theorem, and a new weighted compact Sobolev-type embedding theorem due to Shapiro.

This paper is organized as follows. In Section 2, we introduce some necessary assumptions and basic results. In Section 3, five fundamental lemmas are established. The subsequent Section 4 contains proofs of the main results.

\section{Basic Assumptions and Main Theorem}

In this section, we introduce some assumptions and give the main results in this paper.

Let $\Omega \subset R^{N}(N \geq 1)$ be an open (possibly unbounded) set, $\Gamma \subset \partial \Omega$ a fixed closed set ( $\Gamma$ may be an empty set), $\widetilde{\Omega}=$ $\Omega \times T$, and $T=(-\pi, \pi)$. We assume throughout this paper that $\rho(x), p_{i}(x)(i=1,2, \ldots, N)$ are positive $C^{0}(\Omega)$ functions, function $q(x)$ is a nonnegative $C^{0}(\Omega)$ function $(q(x)$ may be zero), and

$$
\begin{gathered}
\int_{\Omega} \rho(x) d x<\infty, \quad \int_{\Omega} p_{i}(x) d x<\infty, \quad i=1,2, \ldots, N, \\
\exists K>0, \text { s.t. } 0 \leq q(x) \leq K \rho(x), \quad \forall x \in \Omega .
\end{gathered}
$$

We define $p=\left(p_{1}, \ldots, p_{N}\right), D_{i} u=\partial u / \partial x_{i}, i=$ $1,2, \ldots, N$, and $\mathscr{A}$ is a set of real-valued functions defined as

$$
\begin{gathered}
\mathscr{A}=\left\{u: u \in C^{0}(\bar{\Omega} \times R), u(x, t+2 \pi)=u(x, t),\right. \\
\forall(x, t) \in \bar{\Omega} \times R\} .
\end{gathered}
$$

We consider the following pre-Hilbert spaces (see [1]):

$$
\widetilde{C}_{\rho}^{0}(\widetilde{\Omega})=\left\{\left.u \in C^{0}(\widetilde{\Omega})\left|\int_{\widetilde{\Omega}}\right| u(x, t)\right|^{2} \rho(x) d x d t<\infty\right\},
$$

with inner product $\langle u, v\rangle_{\rho}^{\sim}=\int_{\widetilde{\Omega}} u(x, t) v(x, t) \rho(x) d x d t$, and

$$
\begin{aligned}
\widetilde{C}_{p, \rho}^{1}(\widetilde{\Omega}, \Gamma) & \\
= & \left\{u \in \mathscr{A} \cap C^{1}(\Omega \times R) \mid u(x, t)=0,\right. \\
& \forall(x, t) \in \Gamma \times R ; \\
& \left.\int_{\widetilde{\Omega}}\left(\sum_{i=1}^{N}\left|D_{i} u\right|^{2} p_{i}+\left(u^{2}+\left|D_{t} u\right|^{2}\right) \rho\right)<\infty\right\},
\end{aligned}
$$

the two-form of $\mathscr{L}$ is

$$
\mathscr{L}(u, v)=\sum_{i=1}^{N} \int_{\Omega} p_{i} D_{i} u D_{i} v+\int_{\Omega} u v q, \quad u, v \in H_{p, q, \rho}(\Omega, \Gamma),
$$

with inner product

$$
\langle u, v\rangle_{\widetilde{H}}=\int_{\widetilde{\Omega}}\left(\sum_{i=1}^{N} p_{i} D_{i} u D_{i} v+\left(u v+D_{t} u D_{t} v\right) \rho\right) d x d t .
$$

We denote $\widetilde{L}_{\rho}^{2} \triangleq L_{\rho}^{2}(\widetilde{\Omega})$ which is the Hilbert space obtained from the completion of $\widetilde{C}_{\rho}^{0}(\widetilde{\Omega})$ with the norm $\|u\|_{\rho}=$ $\left(\langle u, u\rangle_{\rho}^{\sim}\right)^{1 / 2}$, and denote $\widetilde{H} \triangleq \widetilde{H}(\widetilde{\Omega}, \Gamma)$ which is the Hilbert space obtained from the completion of the space $\widetilde{C}_{p, \rho}(\widetilde{\Omega}, \Gamma)$ with the norm $\|u\|_{\widetilde{H}}=\langle u, u\rangle_{\widetilde{H}}^{1 / 2}$. Similarly, we have $\widetilde{L}_{p_{i}}^{2}(i=$ $1,2, \ldots, N)$ and $\widetilde{L}_{q}^{2}$.

It is assumed throughout the paper that $\sigma_{i}(u)(i=$ $0,1, \ldots, N), b_{i j}(x)(i, j=1,2, \ldots, N)$, and $b_{0}(x)$ meet the following assumptions:

$(\sigma-1) \sigma_{i}(u): \widetilde{H} \rightarrow R$ is weakly sequentially continuous;

$(\sigma-2)$ there are $\eta_{0}, \eta_{1}>0$, s.t. $\eta_{0} \leq \sigma_{i}(u) \leq \eta_{1}$, and $\sigma_{i}(u)$ is measurable, for $u \in \widetilde{H}$;

$(b-1) b_{0}(x), b_{i j}(x) \in C^{0}(\Omega) \bigcap L^{\infty}(\Omega)$, where $b_{i j}(x)=b_{j i}(x)$, for $x \in \Omega$;

$(b-2) b_{0}(x) \geq \varepsilon_{0}>0$, for $x \in \Omega$;

(b-3) there is a constant $c_{0}>0$, for $x \in \Omega$ and $\xi \in R^{N}$, such that

$$
\sum_{i, j=1}^{N} b_{i j}(x) \xi_{i} \xi_{j} \geq c_{0}|\xi|^{2}, \quad|\xi|^{2}=\sum_{i=1}^{N} \xi_{i}^{2}
$$

Definition 1. For the quasilinear differential operator $\widetilde{\mathscr{M}}$, the two-form is

$$
\begin{aligned}
\widetilde{M}(u, v)= & \sum_{i, j=1}^{N} \int_{\widetilde{\Omega}}\left(p_{i}^{1 / 2} p_{j}^{1 / 2} \sigma_{i}^{1 / 2}(u) \sigma_{j}^{1 / 2}(u) b_{i j} D_{j} u D_{i} v\right) \\
& +\int_{\widetilde{\Omega}} q \sigma_{0}(u) b_{0} u v, \quad \text { for } u, v \in \widetilde{H} .
\end{aligned}
$$

Defining

$$
\mathscr{L} u=-\sum_{i=1}^{N} D_{i}\left(p_{i} D_{i} u\right)+q u,
$$

for $u \in H_{p, q, \rho}(\Omega, \Gamma)$ (as described in [5]), and

$$
\widetilde{\mathscr{L}} u=-\sum_{i=1}^{N} D_{i}\left(p_{i} D_{i} u\right)+q u, \quad u \in \widetilde{H},
$$


and the two-form of $\widetilde{\mathscr{L}}$ is

$$
\widetilde{\mathscr{L}}(u, v)=\sum_{i=1}^{N} \int_{\widetilde{\Omega}} p_{i} D_{i} u D_{i} v+\int_{\widetilde{\Omega}} u v q, \quad u, v \in \widetilde{H} .
$$

Definition 2. One says that $\widetilde{\mathscr{M}}$ is $\# \widetilde{H}$-related to $\widetilde{\mathscr{L}}$ if the following condition holds:

$$
\lim _{\|u\|_{\widetilde{H}} \rightarrow \infty} \frac{\widetilde{\mathscr{M}}(u, v)-\widetilde{\mathscr{L}}(u, v)}{\|u\|_{\widetilde{H}}}=0 \text {, uniformly for }\|v\|_{\widetilde{H}} \leq 1 \text {. }
$$

Definition 3. $(\widetilde{\Omega}, \Gamma)$ is a simple $V_{L}(\widetilde{\Omega}, \Gamma)$ if

$\left(V_{L}-1\right)$ there is a complete orthonormal system $\left\{\varphi_{n}\right\}_{n=1}^{\infty}$ in $L_{\rho}^{2}$. Also $\varphi_{n} \in H_{p, q, \rho} \cap C^{2}$ for all $n$;

$\left(V_{L}-2\right)$ there is a sequence of eigenvalues $\left\{\lambda_{n}\right\}_{n=1}^{\infty}$ with $0<$ $\lambda_{1}<\lambda_{2} \leq \lambda_{3} \leq \cdots \leq \lambda_{n} \rightarrow \infty$ such that $\mathscr{L}\left(\varphi_{n}, v\right)=$ $\lambda_{n}\left\langle\varphi_{n}, v\right\rangle_{\rho}$ for all $v \in H_{p, q, \rho}(\Omega, \Gamma)$. Also $\varphi_{1}>0$ in $\Omega$;

$\left(V_{L^{-}} 3\right) \Omega=\Omega_{1} \times \cdots \times \Omega_{N}$, where $\Omega_{i} \subset R$ is an open set for $i=1, \ldots, N$

$\left(V_{L}-4\right)$ for each $\Omega_{i}$, there are positive functions $p_{i}^{*}(x)$, $\rho_{i}^{*}(x) \in C^{0}\left(\Omega_{i}\right)$ satisfying $\int_{\Omega_{i}}\left(p_{i}^{*}(x)+\rho_{i}^{*}(x)\right) d x<\infty$, and $\rho(x)=\rho_{1}^{*}\left(x_{1}\right) \cdots \rho_{N}^{*}\left(x_{N}\right), p_{i}(x)=\rho_{1}^{*}\left(x_{1}\right) \cdots$ $\rho_{i-1}^{*}\left(x_{i-1}\right) p_{i}^{*}\left(x_{i}\right) \rho_{i+1}^{*}\left(x_{i+1}\right) \cdots \rho_{N}^{*}\left(x_{N}\right)$;

$\left(V_{L^{-}}\right)$for each $\Omega_{i}, p_{i}^{*}(x)$, and $\rho_{i}^{*}(x)(i=1, \ldots, N)$, there exist $h_{i} \in C^{0}\left(\Omega_{i}\right) \bigcap L_{\rho_{i}^{*}}^{\theta}\left(\Omega_{i}\right)$ and $y_{i}(t) \in C^{0}(T) \bigcap$ $L^{\theta}(T)$ for $2<\theta<\infty$, such that

$$
\begin{array}{r}
|v(s, t)| \leq h_{i}(s) y_{i}(t)\|v\|_{p_{i}^{*}(s), \rho_{i}^{*}(s)}, \\
\forall v \in C^{1}\left(\Omega_{i} \times T\right), \quad \forall s \in \Omega_{i}, \quad t \in T,
\end{array}
$$

where

$$
\|v\|_{p_{i}^{*}(s), \rho_{i}^{*}(s)}^{2}=\int_{\widetilde{\Omega}_{i}}\left(p_{i}^{*}(s)\left|D_{s} v\right|^{2}+\rho_{i}^{*}(s)\left(v^{2}+\left|D_{t} v\right|^{2}\right)\right) d s d t .
$$

There are many examples to illustrate the simple $V_{L}$ region. One can refer to [1].

Remark 4. We give an example to establish existence results for $\widetilde{\mathscr{M}}$ and $\widetilde{\mathscr{L}}$ which meet Definition 2 . Taking $N>1, \Omega \subset$ $R^{N}$ (a bounded open connected set), and $\Gamma=\partial \Omega$, we set $\rho=$ $q=p_{i}=b_{0}=b_{i j}=1$, for $i=1,2, \ldots, N, \sigma_{i}(u)=\sigma_{i}(u)$, and $\widetilde{\mathscr{L}}=-\sum_{i=1}^{N} D_{i i} u+u$. Then, it is easy to see from [9] that $(\Omega, \Gamma)$ is a simple $V_{L}$ region. We set

$$
\begin{aligned}
& \sigma_{i}(u)=D_{i} u+\frac{D_{i} u}{\left(1+u^{2}+|D u|^{2}\right)^{1 / 2}}, \\
& \sigma_{0}(u)=1+\frac{1}{\left(1+u^{2}+|D u|^{2}\right)^{1 / 2}} .
\end{aligned}
$$

Also, we define

$$
\widetilde{\mathscr{M}}=-\sum_{i=1}^{N} D_{i}\left(\sigma_{i}(u) D_{i} u\right)+\sigma_{0}(u) u
$$

We observe with $|D u|^{2}=\sum_{i=1}^{N}\left|D_{i} u\right|^{2}$ that

$$
\begin{aligned}
& |\widetilde{\mathscr{M}}(u, v)-\widetilde{\mathscr{L}}(u, v)| \\
& \quad=\left|\int_{\widetilde{\Omega}} \frac{1}{1+u^{2}+|D u|^{2}}\left(u v+\sum_{i=1}^{N} D_{i} u D_{i} v\right)\right| \\
& \quad \leq(2 \pi|\Omega|)^{1 / 2}|\widetilde{\mathscr{L}}(v, v)|^{1 / 2} .
\end{aligned}
$$

Clearly, $\widetilde{\mathscr{M}}$ and $\widetilde{\mathscr{L}}$ meet Definition 2 .

Remark 5. If $\widetilde{\mathscr{M}}$ (as (12)) meets $(\sigma-1)-(\sigma-2),(b-1)-(b-3)$, then

$$
\widetilde{M}\left(v, D_{t} v\right)=0, \quad \forall v \in \widetilde{C}_{p, \rho}^{1 b}=\left\{v: v \in \widetilde{C}_{p, \rho}^{1} ; D_{t} v \in \widetilde{C}_{p, \rho}^{1}\right\} .
$$

$f(x, s)$ meets the following conditions:

$(f-1) f(x, s)$ satisfies the Carathéodory conditions;

$(f-2)$ (Superlinear condition) there exists $\theta$ with $2<\theta<$ $2 N /(N-1)$ such that $|f(x, s)| \leq h_{0}(x)+K|s|^{\theta-1}, \forall s \in$ $R$, a.e. $x \in \Omega$, where $h_{0}(x) \in L_{\rho}^{\theta^{*}}$. $K$ is a nonnegative constant and $\theta^{*}=\theta /(\theta-1)$.

Now we state our main results in this paper.

Theorem 6. Assume that $(\widetilde{\Omega}, \Gamma)$ is a simple $V_{L}(\widetilde{\Omega}, \Gamma)$. Let $\mathscr{L}$ given by (13) satisfy the conditions of simple $V_{L}(\widetilde{\Omega}, \Gamma)$, and let $\widetilde{M}$ given by (12) satisfy $(b-1)-(b-3),(\sigma-1)-(\sigma-2)$ and be \# $\widetilde{H}$ related to $\widetilde{\mathscr{L}}$. Suppose that $G \in(\widetilde{H})^{\prime}$ (the dual of $\widetilde{H}$ ) and that $(f-1)-(f-2)$ hold. Suppose furthermore there is a nonnegative function $\widetilde{h}_{0}(x) \in L_{\rho}^{\theta^{*}}$ and a constant $\varepsilon_{0}^{*}>0$ such that

$$
s f(x, s) \leq-\varepsilon_{0}^{*}|s|^{2}+\widetilde{h}_{0}(x)|s|, \quad \forall s \in R \text {, a.e. } x \in \Omega .
$$

Then problem (1) has at least one nontrivial weak solution, that is, $\exists u^{*} \in \widetilde{H}$, such that

$$
\begin{aligned}
& \left\langle D_{t} u^{*}, v\right\rangle_{\rho}^{\sim}+\widetilde{\mathscr{M}}\left(u^{*}, v\right) \\
& \quad=\lambda_{1}\left\langle u^{*}, v\right\rangle_{\rho}^{\sim}+\int_{\widetilde{\Omega}} f\left(x, u^{*}\right) v \rho-G(v), \quad \forall v \in \widetilde{H} .
\end{aligned}
$$

Remark 7. Observing that, for $N=2, f(x, s)=-g(x) s|s|^{5 / 3}-$ $\varepsilon_{0}^{*} s$, where $g(x) \in C^{0}(\Omega) \cap L^{\infty}(\Omega)$ is a positive function and $g(x)$ meets $(f-2)$ and $(24)$.

\section{Preliminary Lemmas}

In this section, we introduce some lemmas. First, we introduce some notions. 
If the conditions of simple $V_{L}(\widetilde{\Omega}, \Gamma)$ hold, we get

$$
\left\{\widetilde{\varphi}_{j k}^{c}\right\}_{j=1, k=0}^{\infty, \infty} \cup\left\{\widetilde{\varphi}_{j k}^{s}\right\}_{j=1, k=1}^{\infty, \infty} \text { is a CONS for } \widetilde{L}_{\rho}^{2} \text {, }
$$

where

$$
\begin{array}{ll}
\text { (i) } \widetilde{\varphi}_{j k}^{c}(x, t)= \begin{cases}\frac{1}{\sqrt{2 \pi}} \varphi_{j}(x), & k=0, j=1,2, \ldots, \\
\frac{1}{\sqrt{\pi}} \varphi_{j}(x) \cos k t, & j, k=1,2, \ldots,\end{cases} \\
\text { (ii) } \widetilde{\varphi}_{j k}^{s}(x, t)=\frac{1}{\sqrt{\pi}} \varphi_{j}(x) \sin k t, \quad j, k=1,2, \ldots
\end{array}
$$

Obviously, both $\widetilde{\varphi}_{j k}^{c}$ and $\widetilde{\varphi}_{j k}^{s}$ are in $\widetilde{H}$. Defining

$$
\mathscr{L}_{1}(u, v)=\widetilde{\mathscr{L}}(u, v)+\langle u, v\rangle_{\rho}^{\sim}, \quad \forall u, v \in \widetilde{H},
$$

it is clear that $\mathscr{L}_{1}(u, v)$ is an inner product on $\widetilde{H}$. From (10), (16), and (28), there are $K_{1}, K_{2}>0$ such that

$$
K_{1}\|v\|_{\widetilde{H}}^{2} \leq \mathscr{L}_{1}(v, v)+\left\|D_{t} v\right\|_{\rho}^{2} \leq K_{2}\|v\|_{\widetilde{H}}^{2}, \quad \forall v \in \widetilde{H} .
$$

For $v \in \widetilde{L}_{\rho}^{2}$, setting

$$
\widehat{v}^{c}(j, k)=\left\langle v, \tilde{\varphi}_{j k}^{c}\right\rangle_{\rho}^{\sim}, \quad \widehat{v}^{s}(j, k)=\left\langle v, \tilde{\varphi}_{j k}^{s}\right\rangle_{\rho}^{\sim},
$$

and from $\left(V_{L^{-}}-2\right),(16)$, and (28), we see that, for $v \in \widetilde{H}$,

$$
\begin{aligned}
& \mathscr{L}_{1}\left(v, \widetilde{\varphi}_{j k}^{s}\right)=\left(\lambda_{j}+1\right) \widehat{v}^{s}(j, k), \\
& \mathscr{L}_{1}\left(v, \widetilde{\varphi}_{j k}^{c}\right)=\left(\lambda_{j}+1\right) \widehat{v}^{c}(j, k) .
\end{aligned}
$$

Lemma 8. If $\left\{\widetilde{\varphi}_{j k}^{c}\right\}_{j=1, k=0}^{\infty, \infty} \cup\left\{\widetilde{\varphi}_{j k}^{s}\right\}_{j=1, k=1}^{\infty, \infty}$ is a CONS for $L_{\rho}^{2}(\widetilde{\Omega})$ defined by (27), setting

$$
\tau_{n}(v)=\sum_{j=1}^{n} \widehat{v}^{c}(j, 0) \tilde{\varphi}_{j 0}^{c}+\sum_{j, k=1}^{n}\left(\widehat{v}^{c}(j, k) \tilde{\varphi}_{j k}^{c}+\widehat{v}^{s}(j, k) \tilde{\varphi}_{j k}^{s}\right),
$$

then

$$
\lim _{n \rightarrow \infty}\left\|\tau_{n}(v)-v\right\|_{\widetilde{H}}=0, \quad \forall v \in \widetilde{H}
$$

Lemma 9. (i) If $v \in \widetilde{H}$, then

$$
\begin{aligned}
\mathscr{L}_{1}(v, v) & +\left\|D_{t} v\right\|_{\rho}^{2} \\
= & \sum_{j=1}^{\infty}\left|\widehat{v}^{c}(j, 0)\right|^{2}\left(\lambda_{j}+1\right) \\
& +\sum_{j, k=1}^{\infty}\left(\left|\widehat{v}^{c}(j, k)\right|^{2}+\left|\widehat{v}^{s}(j, k)\right|^{2}\right)\left(\lambda_{j}+1+k^{2}\right) .
\end{aligned}
$$

Lemma 10. Let $\mathscr{L}$ be as Theorem 6 and assume that $(\widetilde{\Omega}, \Gamma)$ is a simple $V_{L}(\widetilde{\Omega}, \Gamma)$. Then $\widetilde{H}$ is compactly imbedded in $L_{\rho}^{2}(\widetilde{\Omega})$.

For the proofs of Lemmas 8-10, one can refer to [5]. We define

$$
S_{n}=\left\{v \in \widetilde{H}: v=\sum_{j=1}^{n} \eta_{j 0}^{c} \widetilde{\varphi}_{j 0}^{c}+\sum_{j, k=1}^{n} \eta_{j k}^{c} \widetilde{\varphi}_{j k}^{c}+\eta_{j k}^{s} \widetilde{\varphi}_{j k}^{s}\right\} .
$$

Remark 11. If $u_{n} \in S_{n}$, then $\widetilde{\mathscr{M}}\left(u_{n}, D_{t} u_{n}\right)=0$.

Lemma 12. Let $\mathscr{L}$ be given as (13) and suppose that $(\widetilde{\Omega}, \Gamma)$ is a simple $V_{L}(\widetilde{\Omega}, \Gamma)$. Then $\widetilde{H}$ is continuously imbedded in $L_{\rho}^{\theta}(\widetilde{\Omega})$ for every $\theta$ satisfying $2<\theta<2 N /(N-1)$, that is, $\exists K_{\theta}>0$, such that

$$
\left(\int_{\widetilde{\Omega}}|u|^{\theta} \rho d x d t\right)^{1 / \theta} \leq K_{\theta}\|u\|_{\widetilde{H}}, \quad \forall u \in \widetilde{H} .
$$

Proof. To establish the lemma, we need to only prove the case that

$$
\left(\int_{\widetilde{\Omega}}|v|^{\theta} \rho d x d t\right)^{1 / \theta} \leq K_{\theta}\|v\|_{\widetilde{H}}
$$

$$
\text { where } v \in \widetilde{C}_{p, \rho}^{1 b}(\widetilde{\Omega}, \Gamma), \quad \text { for } N \geq 2 \text {. }
$$

For fixed $\left(x_{2}, \ldots, x_{N}\right), v\left(x_{1}, x_{2}, \ldots, x_{N}, t\right)$ is a function only depending on $\left(x_{1}, t\right)$ in $C^{1}\left(\widetilde{\Omega}_{1}\right)$. Hence, with (18) and (19), one gets

$$
\left|v\left(x_{1}, x_{2}, \ldots, x_{N}, t\right)\right| \leq h_{1}\left(x_{1}\right) y_{1}(t) g_{1}^{1 / 2}\left(x_{2}, \ldots, x_{N}\right),
$$

where $g_{1}\left(x_{2}, \ldots, x_{N}\right)=\int_{\widetilde{\Omega}_{1}}\left[\left|D_{1} v(x, t)\right|^{2} p_{1}^{*}\left(x_{1}\right)+\left(|v(x, t)|^{2}+\right.\right.$ $\left.\left.\left|D_{t} v(x, t)\right|^{2}\right) \rho_{1}^{*}\left(x_{1}\right)\right] d x_{1} d t$. Applying the same kind of reasoning to $\left(x_{i}, t\right)$ for fixed $\left(x_{1}, \ldots, x_{i-1}, x_{i+1}, \ldots, x_{N}\right)$, we have

$$
|v(x, t)|^{N} \leq \prod_{i=1}^{N} h_{i}\left(x_{i}\right) y_{i}(t) g_{i}^{1 / 2}\left(\breve{x}_{i}\right),
$$

where

$$
\breve{x}_{i}=\left(x_{1}, \ldots, x_{i-1}, x_{i+1}, \ldots, x_{N}\right)
$$

$$
\begin{aligned}
& g_{i}\left(\breve{x}_{i}\right) \\
& =\int_{\widetilde{\Omega}_{i}}\left[\left|D_{i} v(x, t)\right|^{2} p_{i}^{*}\left(x_{i}\right)\right. \\
& \left.+\left(\left|D_{t} v(x, t)\right|^{2}+|v(x, t)|^{2}\right) \rho_{i}^{*}\left(x_{i}\right)\right] d x_{i} d t .
\end{aligned}
$$

By (39), it follows that

$$
\begin{aligned}
& |v(x, t)|^{\theta} \\
& \leq \prod_{i=1}^{N}\left|y_{i}(t)\right|^{\theta / N} \prod_{i=2}^{N}\left|h_{i}\left(x_{i}\right)\right|^{\theta / N}\left|g_{1}\left(\breve{x}_{1}\right)\right|^{\theta / 2 N}\left|h_{1}\left(x_{1}\right)\right|^{\theta / N} \\
& \quad \times \prod_{i=2}^{N}\left|g_{i}\left(\breve{x}_{i}\right)\right|^{\theta / 2 N}
\end{aligned}
$$

(ii) If $v \in L_{\rho}^{2}(\widetilde{\Omega})$ and $\mathscr{L}_{1}(v, v)+\left\|D_{t} v\right\|_{\rho}^{2}<\infty$, then $v \in \widetilde{H}$. 
with $2<\theta<2 N /(N-1)(N \geq 2)$. Taking $r_{1}=\beta=2 N /(2 N-$ $(N-1) \theta)$ and $r_{2}=\cdots=r_{N}=\gamma=2 N / \theta$ and applying the generalized version of Hölder inequality on $\Omega_{1}$, we deduce

$$
\begin{aligned}
\int_{\Omega_{1}}|v(x, t)|^{\theta} \rho_{1}^{*}\left(x_{1}\right) d x_{1} \\
\leq \prod_{i=1}^{N}\left|y_{i}(t)\right|^{\theta / N} \prod_{i=2}^{N}\left|h_{i}\left(x_{i}\right)\right|^{\theta / N}\left|g_{1}\left(\breve{x}_{1}\right)\right|^{\theta / 2 N} \\
\cdot\left[\int_{\Omega_{1}}\left|h_{1}\left(x_{1}\right)\right|^{\beta \theta / N} \rho_{1}^{*}\left(x_{1}\right) d x_{1}\right]^{1 / \beta} \\
\times \prod_{i=2}^{N}\left[\int_{\Omega_{1}} g_{i}\left(\breve{x}_{i}\right) \rho_{1}^{*}\left(x_{1}\right) d x_{1}\right]^{1 / \gamma}
\end{aligned}
$$

Integrating the last inequality on both sides with respect to $x_{2}$, we get

$$
\begin{aligned}
& \int_{\Omega_{2}} \int_{\Omega_{1}}|v(x, t)|^{\theta} \rho_{1}^{*}\left(x_{1}\right) \rho_{2}^{*}\left(x_{2}\right) d x_{1} d x_{2} \\
& \leq \prod_{i=1}^{N}\left|y_{i}(t)\right|^{\theta / N} \prod_{i=3}^{N}\left|h_{i}\left(x_{i}\right)\right|^{\theta / N}\left[\int_{\Omega_{1}}\left|g_{2}\left(\breve{x}_{2}\right)\right| \rho_{1}^{*}\left(x_{1}\right) d x_{1}\right]^{1 / \gamma} \\
& \quad \cdot \prod_{i=1}^{2}\left[\int_{\Omega_{i}}\left|h_{i}\left(x_{i}\right)\right|^{\beta \theta / N} \rho_{i}^{*}\left(x_{i}\right) d x_{i}\right]^{1 / \beta} \\
& \quad \times\left[\int_{\Omega_{2}}\left|g_{1}\left(\breve{x}_{1}\right)\right| \rho_{2}^{*}\left(x_{2}\right) d x_{2}\right]^{1 / \gamma} \\
& \quad \cdot \prod_{i=3}^{N}\left[\int_{\Omega_{2}} \int_{\Omega_{1}} g_{i}\left(\breve{x}_{i}\right) \rho_{1}^{*}\left(x_{1}\right) \rho_{2}^{*}\left(x_{2}\right) d x_{1} d x_{2}\right]^{1 / \gamma} .
\end{aligned}
$$

We continue integrating with respect to $x_{3}, \ldots, x_{N-1}$ and eventually find

$$
\begin{aligned}
& \int_{\Omega_{N-1}} \int_{\Omega_{N-2}} \cdots \int_{\Omega_{1}}|v(x, t)|^{\theta} \rho_{1}^{*}\left(x_{1}\right) \cdots \rho_{N-2}^{*}\left(x_{N-2}\right) \\
& \times \rho_{N-1}^{*}\left(x_{N-1}\right) d x_{1} \cdots d x_{N-2} d x_{N-1} \\
& \leq \prod_{i=1}^{N}\left|y_{i}(t)\right|^{\theta / N} \\
& \quad \times \prod_{i=1}^{N-1}\left[\int_{\Omega_{i}}\left|h_{i}\left(x_{i}\right)\right|^{\beta \theta / N} \rho_{i}^{*}\left(x_{i}\right) d x_{i}\right]^{1 / \beta}\left|h_{N}\left(x_{N}\right)\right|^{\theta / N} \\
& \quad \cdot\left[\int_{\Omega_{N-2}} \cdots \int_{\Omega_{1}} g_{N-1}\left(\breve{x}_{N-1}\right) \rho_{1}^{*}\left(x_{1}\right)\right. \\
& \left.\cdots \rho_{N-2}^{*}\left(x_{N-2}\right) d x_{1} \cdots d x_{N-2}\right]^{1 / \gamma}
\end{aligned}
$$

$$
\begin{aligned}
& \cdot\left[\int_{\Omega_{N-1}} \int_{\Omega_{N-2}} \cdots \int_{\Omega_{1}} g_{N}\left(\breve{x}_{N}\right) \rho_{1}^{*}\left(x_{1}\right) \cdots \rho_{N-2}^{*}\left(x_{N-2}\right)\right. \\
& \left.\times \rho_{N-1}^{*}\left(x_{N-1}\right) d x_{1} \cdots d x_{N-2} d x_{N-1}\right]^{1 / \gamma} \\
& \cdot\left[\int_{\Omega_{N-1}} \int_{\Omega_{N-3}} \cdots \int_{\Omega_{1}} g_{N-2}\left(\breve{x}_{N-2}\right) \rho_{1}^{*}\left(x_{1}\right) \cdots \rho_{N-3}^{*}\left(x_{N-3}\right)\right. \\
& \left.\times \rho_{N-1}^{*}\left(x_{N-1}\right) d x_{1} \cdots d x_{N-3} d x_{N-1}\right]^{1 / \gamma} \\
& \cdot\left[\int_{\Omega_{N-1}} \cdots \int_{\Omega_{3}} \int_{\Omega_{1}} g_{2}\left(\breve{x}_{2}\right) \rho_{1}^{*}\left(x_{1}\right) \rho_{3}^{*}\left(x_{3}\right) \cdots \rho_{N-1}^{*}\right. \\
& \left.\times\left(x_{N-1}\right) d x_{1} d x_{3} \cdots d x_{N-1}\right]^{1 / \gamma} \\
& \cdot\left[\int_{\Omega_{N-1}} \int_{\Omega_{N-2}} \cdots \int_{\Omega_{3}} \int_{\Omega_{2}} g_{1}\left(\breve{x}_{1}\right)\right. \\
& \times \rho_{2}^{*}\left(x_{2}\right) \rho_{3}^{*}\left(x_{3}\right) \\
& \cdots \rho_{N-2}^{*}\left(x_{N-2}\right) \\
& \times \rho_{N-1}^{*}\left(x_{N-1}\right) d x_{2} d x_{3} \\
& \left.\cdots d x_{N-2} d x_{N-1}\right]^{1 / \gamma} \text {. }
\end{aligned}
$$

Furthermore, we have

$$
\begin{gathered}
\int_{\widetilde{\Omega}_{N}} \int_{\Omega_{N-1}} \int_{\Omega_{N-2}} \cdots \int_{\Omega_{1}}|v(x, t)|^{\theta} \rho_{1}^{*}\left(x_{1}\right) \cdots \rho_{N-2}^{*}\left(x_{N-2}\right) \\
\times \rho_{N-1}^{*}\left(x_{N-1}\right) \rho_{N}^{*}\left(x_{N}\right) d x_{1} \\
\cdots d x_{N-2} d x_{N-1} d x_{N} d t \\
\leq \int_{T} \prod_{i=1}^{N}\left|y_{i}(t)\right|^{\theta / N} d t \\
\quad \times \prod_{i=1}^{N}\left[\int_{\Omega_{i}}\left|h_{i}\left(x_{i}\right)\right|^{\beta \theta / N} \rho_{i}^{*}\left(x_{i}\right) d x_{i}\right]^{1 / \beta}\left[\prod_{i=1}^{N} J_{i}\right]^{1 / \gamma}
\end{gathered}
$$

with

$$
\begin{array}{r}
J_{i}=\int_{\Omega_{N}} \cdots \int_{\Omega_{i+1}} \int_{\Omega_{i-1}} \cdots \int_{\Omega_{1}} g_{i}\left(\breve{x}_{i}\right) \rho_{1}^{*}\left(x_{1}\right) \\
\cdots \rho_{i-1}^{*}\left(x_{i-1}\right) \rho_{i+1}^{*}\left(x_{i+1}\right) \\
\cdots \rho_{N}^{*}\left(x_{N}\right) d x_{1} \\
\cdots d x_{i-1} d x_{i+1} \cdots d x_{N} .
\end{array}
$$


From (18) we see that $h_{i} \in C^{0}\left(\Omega_{i}\right) \cap L_{\rho_{i}^{*}}^{\theta}\left(\Omega_{i}\right)$ and $y_{i}(t) \epsilon$ $C^{0}(T) \cap L^{\theta}(T)$. Hence, we obtain from (46) that there is a constant $K_{\theta}$ such that

$$
\left(\int_{\widetilde{\Omega}}|v(x, t)|^{\theta} \rho(x) d x d t\right)^{1 / \theta} \leq K_{\theta}\left(\prod_{i=1}^{N} J_{i}\right)^{1 / 2 N} .
$$

By $(41),(47)$, and $p_{i}(x)=\rho_{1}^{*}\left(x_{1}\right) \cdots \rho_{i-1}^{*}\left(x_{i-1}\right) p_{i}^{*}\left(x_{i}\right) \rho_{i+1}^{*}\left(x_{i+1}\right)$ $\cdots \rho_{N}^{*}\left(x_{N}\right)$, we see that

$$
\begin{aligned}
J_{i}=\int_{\widetilde{\Omega}}\left[\left|D_{i} v(x, t)\right|^{2} p_{i}(x)\right. \\
\left.\quad+\left(\left|D_{t} v(x, t)\right|^{2}+|v(x, t)|^{2}\right) \rho(x)\right] d x d t .
\end{aligned}
$$

Therefore $J_{i} \leq\|v\|_{\widetilde{H}}^{2}$ for $i=1, \ldots, N$, and $\prod_{i=1}^{N} J_{i} \leq\|v\|_{\widetilde{H}}^{2 N}$.

By using (48), we get (36). The proof of Lemma 12 is complete.

Lemma 13. Let $\mathscr{L} u$ be given by (13) and suppose that $(\widetilde{\Omega}, \Gamma)$ is a simple $V_{L}(\widetilde{\Omega}, \Gamma)$. Then, for $N \geq 2, \widetilde{H}$ is compactly imbedded in $L_{\rho}^{\theta}(\widetilde{\Omega})$ for $2<\theta<2 N /(N-1)$. For $N=1, \widetilde{H}$ is compactly imbedded in $L_{\rho}^{\theta}(\widetilde{\Omega})$ for $2<\theta<\infty$.

Lemma 13 is an immediate byproduct of [1, Theorem 9].

\section{Proof of Theorem 6}

In this section, we will give the proof of Theorem 6 . In order to do this, we divide the proof into three parts.

Lemma 14. Let all the assumptions in Theorem 6 hold. Then, for $n \geq 2, \exists u_{n} \in S_{n}$ such that

$$
\begin{aligned}
& \left\langle D_{t} u_{n}, v\right\rangle_{\rho}^{\sim}+\widetilde{\mathscr{M}}\left(u_{n}, v\right) \\
& =\left(\lambda_{1}-\frac{1}{n}\right)\left\langle u_{n}, v\right\rangle_{\rho}^{\sim}+\left\langle f\left(x, u_{n}\right), v\right\rangle_{\rho}^{\sim}-G(v), \quad \forall v \in S_{n} .
\end{aligned}
$$

Proof. To prove the lemma, we first observe from (35) that

(i) $v \in S_{n} \Longrightarrow D_{t} v \in S_{n}$,

(ii) $\left\langle D_{t}\left(\alpha \tilde{\varphi}_{j k}^{c}+\beta \tilde{\varphi}_{j k}^{s}\right), \alpha \widetilde{\varphi}_{j k}^{c}+\beta \widetilde{\varphi}_{j k}^{s}\right\rangle_{\rho}^{\sim}=0$,

$$
\text { for } j, k \geq 1, \quad \alpha, \beta \in R \text {. }
$$

Let $\left\{\psi_{i}\right\}_{i=1}^{2 n^{2}+n}$ be an enumeration of $\left\{\widetilde{\varphi}_{j k}^{c}\right\}_{j=1, k=0}^{n, n} \cup$ $\left\{\widetilde{\varphi}_{j k}^{s}\right\}_{j=1, k=1}^{n, n}$, and set

$$
n^{*}=\left(j_{0}+j_{1}-1\right)(2 n+1) .
$$

So $\left\{\psi_{i}\right\}_{i=1}^{n^{*}}$ is an enumeration of $\left\{\widetilde{\varphi}_{j k}^{c}\right\}_{j=1, k=0}^{j_{0}+j_{1}-1, n} \cup\left\{\widetilde{\varphi}_{j k}^{s}\right\}_{j=1, k=1}^{j_{0}+j_{1}-1, n}$, where $n \geq n_{0}$.

For $\alpha=\left(\alpha_{1}, \ldots, \alpha_{2 n^{2}+n}\right)$, setting

$$
u=\sum_{i=1}^{2 n^{2}+n} \alpha_{i} \psi_{i}, \quad \widetilde{u}=\sum_{i=1}^{2 n^{2}+n} \delta_{i} \alpha_{i} \psi_{i},
$$

where

$$
\delta_{i}= \begin{cases}-1, & 1 \leq i \leq n^{*} \\ 1, & n^{*}+1 \leq i \leq 2 n^{2}+n,\end{cases}
$$

from (52)-(54), we have

$$
\begin{gathered}
\tilde{u}=\sum_{j=1}^{n} \delta_{j} \widehat{u}^{c}(j, 0) \widetilde{\varphi}_{j 0}^{c}+\sum_{j, k=1}^{n} \delta_{j}\left[\widehat{u}^{c}(j, k) \widetilde{\varphi}_{j k}^{c}+\widehat{u}^{s}(j, k) \widetilde{\varphi}_{j k}^{s}\right], \\
u=\sum_{j=1}^{n} \widehat{u}^{c}(j, 0) \widetilde{\varphi}_{j 0}^{c}+\sum_{j, k=1}^{n}\left[\widehat{u}^{c}(j, k) \widetilde{\varphi}_{j k}^{c}+\widehat{u}^{s}(j, k) \widetilde{\varphi}_{j k}^{s}\right], \\
\delta_{j}= \begin{cases}-1, & 1 \leq j \leq j_{0}+j_{1}-1, \\
1, & j_{0}+j_{1} \leq j \leq n,\end{cases} \\
=\sum_{j=1}^{n}\left|\widehat{u}^{c}(j, 0)\right|^{2}(\lambda,+1) \\
+\sum_{j=1}^{n} \sum_{k=1}^{n}\left[\left|\widehat{u}^{c}(j, k)\right|^{2}+\left|\widehat{u}^{s}(j, k)\right|^{2}\right]\left(\lambda_{j}+1+k^{2}\right) .
\end{gathered}
$$

Further, (29) yields

$$
\begin{aligned}
K_{1}\|u\|_{\widetilde{H}}^{2} \leq & \mathscr{L}_{1}(u, u)+\left\|D_{t} u\right\|_{\rho}^{2} \\
\leq & \sum_{j=1}^{n}\left|\widehat{u}^{c}(j, 0)\right|^{2}\left(\lambda_{n}+1\right) \\
& +\sum_{j, k=1}^{n}\left[\left|\widehat{u}^{c}(j, k)\right|^{2}+\left|\widehat{u}^{s}(j, k)\right|^{2}\right]\left(\lambda_{n}+1+n^{2}\right) \\
\leq & \left(\lambda_{n}+1+n^{2}\right)\|u\|_{\rho}^{2}, \quad \forall u \in S_{n} .
\end{aligned}
$$

For $m \geq 2$, we set

$$
f_{m}(x, s)= \begin{cases}f(x, m), & \text { for } m \leq s, \\ f(x, s), & \text { for }-m \leq s \leq m, \\ f(x, m), & \text { for } s \leq-m,\end{cases}
$$

and we claim that $\exists u_{m}^{*} \in S_{n}$ such that

$$
\begin{aligned}
& \left\langle D_{t} u_{m}^{*}, v\right\rangle_{\rho}^{\sim}+\widetilde{\mathscr{M}}\left(u_{m}^{*}, v\right) \\
& =\left(\lambda_{1}-\frac{1}{n}\right)\left\langle u_{m}^{*}, v\right\rangle_{\rho}^{\sim}+\int_{\widetilde{\Omega}} f_{m}\left(x, u_{m}^{*}\right) v \rho-G(v), \quad \forall v \in S_{n} .
\end{aligned}
$$

By $(f-2)$ and the above,

$$
\begin{array}{r}
\left|f_{m}(x, s)\right| \leq h_{0}(x)+K|m|^{\theta-1}, \\
\forall s \in R, \quad \text { a.e. } x \in \Omega,
\end{array}
$$


where $h_{0}(x) \in L_{\rho}^{\theta^{*}}$ and $\theta^{*}=\theta /(\theta-1)$. Therefore, $f_{m}(x, u) \epsilon$ $L_{\rho}^{\theta^{*}}(\widetilde{\Omega})$ and $\int_{\widetilde{\Omega}} f_{m}(x, u) v \rho$ is well defined for $v \in S_{n}$ by Lemma 12.

To establish (58), we set

$$
\begin{aligned}
F_{i}(\alpha)= & \left\langle D_{t} u, \psi_{i}\right\rangle_{\rho}^{\sim}+\widetilde{\mathscr{M}}\left(u, \psi_{i}\right) \\
& -\left(\lambda_{1}-\frac{1}{n}\right)\left\langle u, \psi_{i}\right\rangle_{\rho}^{\sim}-\left\langle f_{m}(x, u), \psi_{i}\right\rangle_{\rho}^{\sim}+G\left(\psi_{i}\right)
\end{aligned}
$$

for $i=1, \ldots, n$, and we observe from (51) that

$$
\sum_{i=1}^{2 n^{2}+n} F_{i}(\alpha) \alpha_{i}=I(\alpha)+I I(\alpha)
$$

where

$$
\begin{gathered}
I(\alpha)=\widetilde{\mathscr{L}}(u, u)-\left(\lambda_{1}-\frac{1}{n}\right)\langle u, u\rangle_{\rho}^{\sim}, \\
I I(\alpha)=\widetilde{\mathscr{M}}(u, u)-\widetilde{\mathscr{L}}(u, u)-\int_{\widetilde{\Omega}} f_{m}(x, u) u \rho+G(u) .
\end{gathered}
$$

Now, from (62),

$$
I(\alpha)=\sum_{i=1}^{2 n^{2}+n}\left(\lambda_{i}-\lambda_{1}\right) \alpha_{i}^{2}+\frac{|\alpha|^{2}}{n},
$$

and according to Lemma 12, (56), (59), (63), and the fact that $\widetilde{\mathscr{M}}$ is $\# \widetilde{H}$-related to $\widetilde{\mathscr{L}}$,

$$
\lim _{|\alpha| \rightarrow \infty} \frac{|I I(\alpha)|}{|\alpha|^{2}}=0 .
$$

We see from (61) that $\exists s_{0}>0$ such that

$$
\sum_{i=1}^{2 n^{2}+n} F_{i}(\alpha) \alpha_{i} \geq \frac{|\alpha|^{2}}{2 n}, \quad \text { for }|\alpha|>s_{0} .
$$

Therefore, by virtue of generalized Brouwer's theorem [10], there exists $\alpha^{*}=\left(\alpha_{1}^{*}, \ldots, \alpha_{2 n^{2}+n}^{*}\right)$ such that $F_{i}\left(\alpha^{*}\right)=0$, for $i=1, \ldots, n$. We set $u_{m}^{*}=\sum_{i=1}^{2 n^{2}+n} \alpha_{i}^{*} \psi_{i}$ and see from (60) that (58) does indeed hold.

By the definition of $f_{m}(x, s)$ and (24), for $m \geq 2$,

$$
s f_{m}(x, s) \leq \tilde{h}_{0}(x)|s|, \quad \forall s \in R \text {, a.e. } x \in \Omega,
$$

where $\widetilde{h}_{0}(x) \in L_{\rho}^{\theta^{*}}(\Omega)$.

We claim

$$
\exists K_{1}>0 \text { s.t. }\left\|u_{m}^{*}\right\|_{\rho} \leq K_{1}, \quad \forall m>2 .
$$

Suppose that (68) is false. Then there is a subsequence (which for ease of notation, we take to be the full sequence) such that

$$
\lim _{|m| \rightarrow \infty}\left\|u_{m}^{*}\right\|_{\rho}=\infty
$$

Inserting $u_{m}^{*}$ in place of $v$ in (58), we find

$$
\begin{aligned}
& \widetilde{\mathscr{L}}\left(u_{m}^{*}, u_{m}^{*}\right)-\left(\lambda_{1}-\frac{1}{n}\right)\left\langle u_{m}^{*}, u_{m}^{*}\right\rangle_{\rho}^{\sim} \\
& =\int_{\widetilde{\Omega}} f_{m}\left(x, u_{m}^{*}\right) u_{m}^{*} \rho-G\left(u_{m}^{*}\right)+\widetilde{\mathscr{L}}\left(u_{m}^{*}, u_{m}^{*}\right)-\widetilde{\mathscr{M}}\left(u_{m}^{*}, u_{m}^{*}\right),
\end{aligned}
$$

and the left-hand side of (70) is

$$
\begin{aligned}
& \sum_{j=1}^{n}\left|\widehat{u}_{m}^{* c}(j, 0)\right|^{2}\left(\lambda_{j}-\lambda_{1}\right) \\
& \quad+\sum_{j, k=1}^{n}\left(\left|\widehat{u}_{m}^{* c}(j, k)\right|^{2}+\left|\widehat{u}_{m}^{* s}(j, k)\right|^{2}\right)\left(\lambda_{j}-\lambda_{1}\right)+\frac{\left\|u_{m}^{*}\right\|_{\rho}^{2}}{n} .
\end{aligned}
$$

So we see from (67) and Hölder inequality that

$$
\begin{aligned}
\frac{\left\|u_{m}^{*}\right\|_{\rho}^{2}}{n} \leq & \left\|\widetilde{h}_{0}\right\|_{L_{\rho}^{\theta^{*}}}\left\|u_{m}^{*}\right\|_{L_{\rho}^{\theta}}+\left|G\left(u_{m}^{*}\right)\right| \\
& +\left|\widetilde{\mathscr{L}}\left(u_{m}^{*}, u_{m}^{*}\right)-\widetilde{\mathscr{M}}\left(u_{m}^{*}, u_{m}^{*}\right)\right| .
\end{aligned}
$$

From (36), (56), (69), and the fact that $\widetilde{\mathscr{M}}$ is $\# \widetilde{H}$-related to $\widetilde{\mathscr{L}}$, dividing both sides of (72) by $\left\|u_{m}^{*}\right\|_{\rho}^{2}$ and taking the limit as $m \rightarrow \infty$, we obtain that $1 / n \leq 0$. But $n$ is a positive integer. So we get a contradiction. Therefore, (68) does indeed hold.

Hence, from (56), (68), and $u_{m}^{*} \in S_{n}$, for $m \geq 2$, there is a subsequence $\left\{u_{m_{j}}^{*}\right\}_{j=1}^{\infty}$ and a $u_{n} \in S_{n}$ [11] such that

$$
\begin{gathered}
\lim _{j \rightarrow \infty}\left\|u_{m_{j}}^{*}-u_{n}\right\|_{\widetilde{H}}=0 ; \\
\lim _{j \rightarrow \infty} u_{m_{j}}^{*}(x, t)=u_{n}(x, t), \quad \text { a.e. }(x, t) \in \widetilde{\Omega} ; \\
\lim _{j \rightarrow \infty} D_{i} u_{m_{j}}^{*}(x, t)=D_{i} u_{n}(x, t), \\
\text { a.e. }(x, t) \in \widetilde{\Omega}, \quad i=1, \ldots, N .
\end{gathered}
$$

It is easy to check that $\widetilde{M}\left(u_{m_{j}}^{*}, v\right) \rightarrow \widetilde{M}\left(u_{n}, v\right)$ as $j \rightarrow \infty$, $\forall v \in S_{n}$, and from Lemma 12 we obtain that $\lim _{j \rightarrow \infty} \int_{\widetilde{\Omega}} \mid u_{m_{j}}^{*}-$ $\left.u_{n}\right|^{\theta} \rho=0$. Consequently, there exist a function $W \in L_{\rho}^{\theta}(\widetilde{\Omega})$ and a subsequence $\left\{u_{m_{j_{k}}}^{*}\right\}_{k=1}^{\infty}$ such that

$$
\begin{gathered}
\left|u_{m_{j_{k}}}^{*}(x, t)\right| \leq W(x, t) \quad \text { a.e. }(x, t) \in \widetilde{\Omega}, \forall k, \\
\lim _{k \rightarrow \infty} f_{m_{j_{k}}}\left(x, u_{m_{j_{k}}}^{*}\right)=f\left(x, u_{n}\right) \quad \text { a.e. }(x, t) \in \widetilde{\Omega} .
\end{gathered}
$$

By using $(f-2)$, Hölder inequality, and the Lebesgue dominated convergence theorem, we conclude that

$$
\lim _{k \rightarrow \infty} \int_{\widetilde{\Omega}} f_{m_{j_{k}}}\left(x, u_{m_{j_{k}}}^{*}\right) v \rho=\int_{\widetilde{\Omega}} f\left(x, u_{n}\right) v \rho, \quad \forall v \in S_{n} .
$$


Hence replacing $u_{m}^{*}$ by $u_{m_{j_{k}}}^{*}$ in (58) and passing to the limit as $k \rightarrow \infty$, we consequently obtain that

$$
\begin{aligned}
& \left\langle D_{t} u_{n}, v\right\rangle_{\rho}^{\sim}+\widetilde{M}\left(u_{n}, v\right) \\
& \quad=\left(\lambda_{1}-\frac{1}{n}\right)\left\langle u_{n}, v\right\rangle_{\rho}^{\sim}+\left\langle f\left(x, u_{n}\right), v\right\rangle_{\rho}^{\sim}-G(v), \quad \forall v \in S_{n},
\end{aligned}
$$

and the proof of the lemma is complete.

Lemma 15. Let the conditions in Lemma 14 hold. Then the sequence $\left\{u_{n}\right\}$ obtained in Lemma 14 is uniformly bounded in $\widetilde{H}$ with respect to the norm $\left\|u_{n}\right\|_{\widetilde{H}}=\left\langle u_{n}, u_{n}\right\rangle_{\widetilde{H}}^{1 / 2}$.

Proof. According to Lemma 14, we obtain a sequence $\left\{u_{n}\right\}_{n=2}^{\infty}$, where

$$
u_{n}=\sum_{i=1}^{2 n^{2}+n} \alpha_{(i, n)} \psi_{i}
$$

and $u_{n}$ satisfies

$$
\begin{aligned}
& \left\langle D_{t} u_{n}, v\right\rangle_{\rho}^{\sim}+\widetilde{\mathscr{M}}\left(u_{n}, v\right) \\
& =\left(\lambda_{1}-\frac{1}{n}\right)\left\langle u_{n}, v\right\rangle_{\rho}^{\sim}+\left\langle f\left(x, u_{n}\right), v\right\rangle_{\rho}^{\sim}-G(v), \quad \forall v \in S_{n} .
\end{aligned}
$$

We claim there is a constant $K$ such that

$$
\left\|u_{n}\right\|_{\widetilde{H}} \leq K, \quad \forall n .
$$

Suppose that the assertion is false. Then, without loss of generality, we assume

$$
\lim _{n \rightarrow \infty}\left\|u_{n}\right\|_{\widetilde{H}}=\infty
$$

Putting $u_{n}$ in place of $v$ in (78), we have

$$
\begin{aligned}
& \left\langle D_{t} u_{n}, u_{n}\right\rangle_{\rho}^{\sim}+\widetilde{\mathscr{M}}\left(u_{n}, u_{n}\right) \\
& =\left(\lambda_{1}-\frac{1}{n}\right)\left\langle u_{n}, u_{n}\right\rangle_{\rho}^{\sim}+\left\langle f\left(x, u_{n}\right), u_{n}\right\rangle_{\rho}^{\sim}-G\left(u_{n}\right) .
\end{aligned}
$$

Next, we observe from (77) and Lemma 8 that

$$
\begin{aligned}
\widetilde{\mathscr{L}}\left(u_{n}, u_{n}\right)= & \sum_{j=1}^{n}\left|\widehat{u}_{n}^{c}(j, 0)\right|^{2} \lambda_{j} \\
& +\sum_{j, k=1}^{n}\left(\left|\widehat{u}_{n}^{c}(j, k)\right|^{2}+\left|\widehat{u}_{n}^{s}(j, k)\right|^{2}\right) \lambda_{j} \\
\geq & \lambda_{1}\left\|u_{n}\right\|_{\rho}^{2}
\end{aligned}
$$

and from (78), (24), and Hölder inequality that

$$
\begin{aligned}
\varepsilon_{0}^{*}\left\|u_{n}\right\|_{\rho}^{2} \leq & \left\|\widetilde{h}_{0}\right\|_{\widetilde{L}_{\rho}^{\theta^{*}}}\left\|u_{n}\right\|_{\tilde{L}_{\rho}^{\theta}}+\left|G\left(u_{n}\right)\right| \\
& +\left|\widetilde{\mathscr{L}}\left(u_{n} u_{n}\right)-\widetilde{\mathscr{M}}\left(u_{n}, u_{n}\right)\right| .
\end{aligned}
$$

Hence it follows from Lemma $12, G \in(\widetilde{H})^{\prime}, \widetilde{\mathscr{M}}$ being $\# \widetilde{H}$ related to $\widetilde{\mathscr{L}}, \varepsilon_{0}^{*}$ being strictly positive, and (83) that

$$
\lim _{n \rightarrow \infty} \frac{\left\|u_{n}\right\|_{\rho}}{\left\|u_{n}\right\|_{\widetilde{H}}}=0 .
$$

Next, we observe from (12) and (16) that there is a positive constant $c$ such that

$$
c \widetilde{\mathscr{L}}\left(u_{n}, u_{n}\right) \leq \widetilde{\mathscr{M}}\left(u_{n}, u_{n}\right), \quad \forall n
$$

Putting $D_{t} u_{n}$ for $v$ in (78), we see that there exists $K>0$ such that

$$
\left\|D_{t} u_{n}\right\|_{\rho} \leq K
$$

But then it follows from (81), (24), and $\left\|D_{t} u_{n}\right\|_{\rho} \leq K$ that

$$
\begin{aligned}
& c \widetilde{\mathscr{L}}\left(u_{n}, u_{n}\right)+\varepsilon_{0}^{*}\left\|u_{n}\right\|_{\rho}^{2}+\left\|D_{t} u_{n}\right\|_{\rho}^{2} \\
& \leq\left\|\widetilde{h}_{0}\right\|_{\widetilde{L}_{\rho}^{\theta^{*}}}\left\|u_{n}\right\|_{\tilde{L}_{\rho}^{\theta}}+\lambda_{1}\left\|u_{n}\right\|_{\rho}^{2}+\left|G\left(u_{n}\right)\right|+K .
\end{aligned}
$$

So, we see from (28), (29), and (87) that there is a positive constant $K_{1}$ such that

$$
\left\|u_{n}\right\|_{\widetilde{H}}^{2} \leq K_{1}\left(\left\|\widetilde{h}_{0}\right\|_{\widetilde{L}_{\rho}^{\theta^{*}}}\left\|u_{n}\right\|_{\widetilde{L}_{\rho}^{\theta}}+\lambda_{1}\left\|u_{n}\right\|_{\rho}^{2}+\left|G\left(u_{n}\right)\right|+K\right) .
$$

Consequently, dividing both sides of (88) by $\left\|u_{n}\right\|_{\widetilde{H}}^{2}$ and passing to the limit as $n \rightarrow \infty$, from Lemma 12, the fact that $G \in(\widetilde{H})^{\prime},(80)$, and (84), we obtain that $1 \leq 0$. This is a contradiction. Hence (80) does not hold and (79) is true.

Proof of Theorem 6. Since $\widetilde{H}$ is a separable Hilbert space, we see from (79) and Lemmas 9 and 13 that there exist a subsequence (for the sake of simplicity, we take to be a full sequence) and a function $u^{*} \in \widetilde{H}$ with the following properties [11]:

(1) $\lim _{n \rightarrow \infty}\left(\left\|u_{n}-u^{*}\right\|_{\rho}+\int_{\widetilde{\Omega}}\left|u_{n}-u^{*}\right|^{\theta} \rho\right)=0$;

(2) $\exists w(x, t) \in \widetilde{L}_{\rho}^{2} \cap \widetilde{L}_{\rho}^{\theta}$, s.t. $\left|u_{n}(x, t)\right| \leq w(x, t)$, a.e. $(x, t) \in \widetilde{\Omega}, \quad \forall n$;

(3) $\lim _{n \rightarrow \infty} u_{n}(x, t)=u^{*}(x, t), \quad$ a.e. $(x, t) \in \widetilde{\Omega}$;

(4) $\lim _{n \rightarrow \infty}\left\langle D_{i} u_{n}, v\right\rangle_{p_{i}}^{\sim}=\left\langle D_{i} u^{*}, v\right\rangle_{p_{i}}^{\sim}$,

$\forall v \in \widetilde{L}_{p_{i}}^{2}, \quad i=1, \ldots, N$

(5) $\lim _{n \rightarrow \infty}\left\langle u_{n}, v\right\rangle_{q}^{\sim}=\left\langle u^{*}, v\right\rangle_{q}^{\sim}, \quad \forall v \in \widetilde{L}_{q}^{2}$;

(6) $\lim _{n \rightarrow \infty} G\left(u_{n}\right)=G\left(u^{*}\right)$;

(7) $\lim _{n \rightarrow \infty}\left\langle D_{t} u_{n}, v\right\rangle_{\rho}^{\sim}=\left\langle D_{t} u^{*}, v\right\rangle_{\rho}^{\sim}, \quad \forall v \in \widetilde{L}_{\rho}^{2}$. 
We let $v_{J} \in S_{J}$. Then it follows from (78) that, for $n \geq J$,

$$
\begin{aligned}
& \left\langle D_{t} u_{n}, v_{J}\right\rangle_{\rho}^{\sim}+\widetilde{\mathscr{M}}\left(u_{n}, v_{J}\right) \\
& =\left(\lambda_{1}-\frac{1}{n}\right)\left\langle u_{n}, v_{J}\right\rangle_{\rho}^{\sim}+\left\langle f\left(x, u_{n}\right), v_{J}\right\rangle_{\rho}^{\sim}-G\left(v_{J}\right) .
\end{aligned}
$$

Now, from $(\sigma-1)-(\sigma-2)$ and $(89)(1)(4)(7)$, we obtin that

$$
\begin{aligned}
\lim _{n \rightarrow \infty} & \left\langle D_{t} u_{n}, v_{J}\right\rangle_{\rho}^{\sim}+\widetilde{\mathscr{M}}\left(u_{n}, v_{J}\right) \\
& =\left\langle D_{t} u_{n}, v_{J}\right\rangle_{\rho}^{\sim}+\widetilde{\mathscr{M}}\left(u_{n}, v_{J}\right) .
\end{aligned}
$$

Next, we observe from $(f-2)$ and (89)(2) that

$$
\begin{array}{r}
\left|f\left(x, u_{n}\right)\right| \leq K|w(x, t)|^{\theta-1}+h_{0}(x) \\
\text { a.e. }(x, t) \in \widetilde{\Omega}, \quad n=1,2, \ldots,
\end{array}
$$

where $w \in \widetilde{L}_{\rho}^{\theta}$ and $h_{0} \in L_{\rho}^{\theta^{*}}$. From $(f-1)$ and (89)(3), we further obtain that

$$
\lim _{n \rightarrow \infty} f\left(x, u_{n}(x, t)\right)=f\left(x, u^{*}(x, t)\right) \quad \text { a.e. }(x, t) \in \widetilde{\Omega} \text {. }
$$

Note that $|w|^{\theta-1} \in \widetilde{L}_{\rho}^{\theta^{*}}$. Hence we conclude from Hölder inequality, the Lebesgue dominated convergence theorem, Lemma 12, and (92) that

$$
\lim _{n \rightarrow \infty} \int_{\widetilde{\Omega}} f\left(x, u_{n}(x, t)\right) v_{J} \rho=\int_{\widetilde{\Omega}} f\left(x, u^{*}(x, t)\right) v_{J} \rho
$$

Then passing to the limit on both sides of (90), we obtain from (91), (94), and (89)(1) that

$$
\begin{aligned}
& \left\langle D_{t} u^{*}, v_{J}\right\rangle_{\rho}+\widetilde{\mathscr{M}}\left(u^{*}, v_{J}\right) \\
& =\lambda_{1}\left\langle u^{*}, v_{J}\right\rangle_{\rho}+\int_{\widetilde{\Omega}} f\left(x, u^{*}(x, t)\right) v_{J} \rho-G\left(v_{J}\right) .
\end{aligned}
$$

Next, for given $v \in \widetilde{H}$, we replace $v_{J}$ with $\tau_{J}(v)$ (defined as (32)) in (95). From (29) and Lemma 8, $\left\|\tau_{J}(v)-v\right\|_{\widetilde{H}} \rightarrow 0$ as $J \rightarrow \infty$. From (95) and Lemma 12, it is then an easy matter to obtain that

$$
\begin{aligned}
\left\langle D_{t} u^{*}, v\right\rangle_{\rho}^{\sim}+\widetilde{M} & \left(u^{*}, v\right) \\
= & \lambda_{1}\left\langle u^{*}, v\right\rangle_{\rho}^{\sim}+\left\langle f\left(x, u^{*}\right), v\right\rangle_{\rho}^{\sim} \\
& -G(v), \quad \forall v \in \widetilde{H},
\end{aligned}
$$

and the proof of Theorem 6 is established.

\section{Conflict of Interests}

The authors declare that there is no conflict of interests regarding the publication of this paper.

\section{Acknowledgments}

This research is supported by the National Natural Science Foundation of China (11171220) and Shanghai Leading Academic Discipline Project (XTKX2012).

\section{References}

[1] V. L. Shapiro, Singular Quasilinearity and Higher Eigenvalues, vol. 726, Memoirs of the American Mathematical Society, Providence, RI, USA, 2001.

[2] A. J. Rumbos and V. L. Shapiro, "Jumping nonlinearities and weighted Sobolev spaces," Journal of Differential Equations, vol. 214, no. 2, pp. 326-357, 2005.

[3] H. Berestycki and D. G. de Figueiredo, "Double resonance in semilinear elliptic problems," Communications in Partial Differential Equations, vol. 6, no. 1, pp. 91-120, 1981.

[4] G. Jia and Q. Zhao, "Existence results in weighted Sobolev spaces for some singular quasilinear elliptic equations," Acta Applicandae Mathematicae, vol. 109, no. 2, pp. 599-607, 2010.

[5] V. L. Shapiro, "Resonance, distributions and semilinear elliptic partial differential equations," Nonlinear Analysis: Theory, Methods \& Applications, vol. 8, no. 8, pp. 857-871, 1984.

[6] A. Rumbos, "A semilinear elliptic boundary value problem at resonance where the nonlinearity may grow linearly," Nonlinear Analysis: Theory, Methods \& Applications, vol. 16, no. 12, pp. 1159-1168, 1991.

[7] L. E. Lefton and V. L. Shapiro, "Resonance and quasilinear parabolic partial differential equations," Journal of Differential Equations, vol. 101, no. 1, pp. 148-177, 1993.

[8] C.-C. Kuo, "On the solvability of a quasilinear parabolic partial differential equation at resonance," Journal of Mathematical Analysis and Applications, vol. 275, no. 2, pp. 913-937, 2002.

[9] D. Gilbarg and N. S. Trudinger, Elliptial Differential Equations of Second Order, Springer, Berlin, Germany, 2nd edition, 1983.

[10] S. Kesavan, Topic in Functional Analysis and Applications, John Wiley \& Sons, New York, NY, USA, 1989.

[11] B. J. Xuan, Variational Methods, University of Science and Technology of China Press, Hefei, China, 2006. 


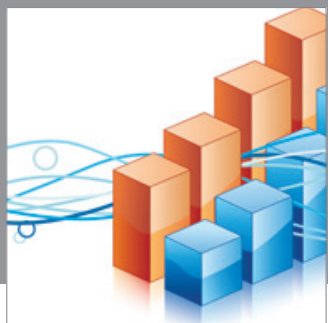

Advances in

Operations Research

mansans

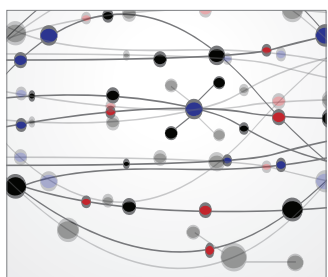

The Scientific World Journal
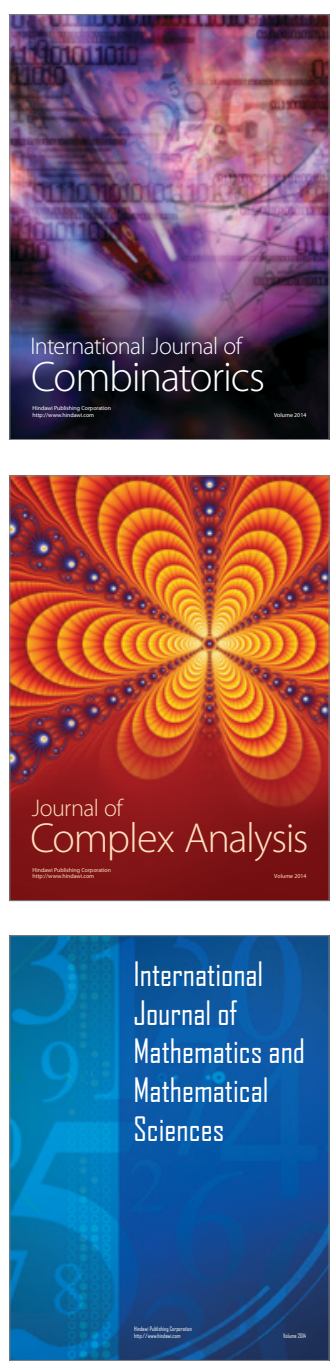
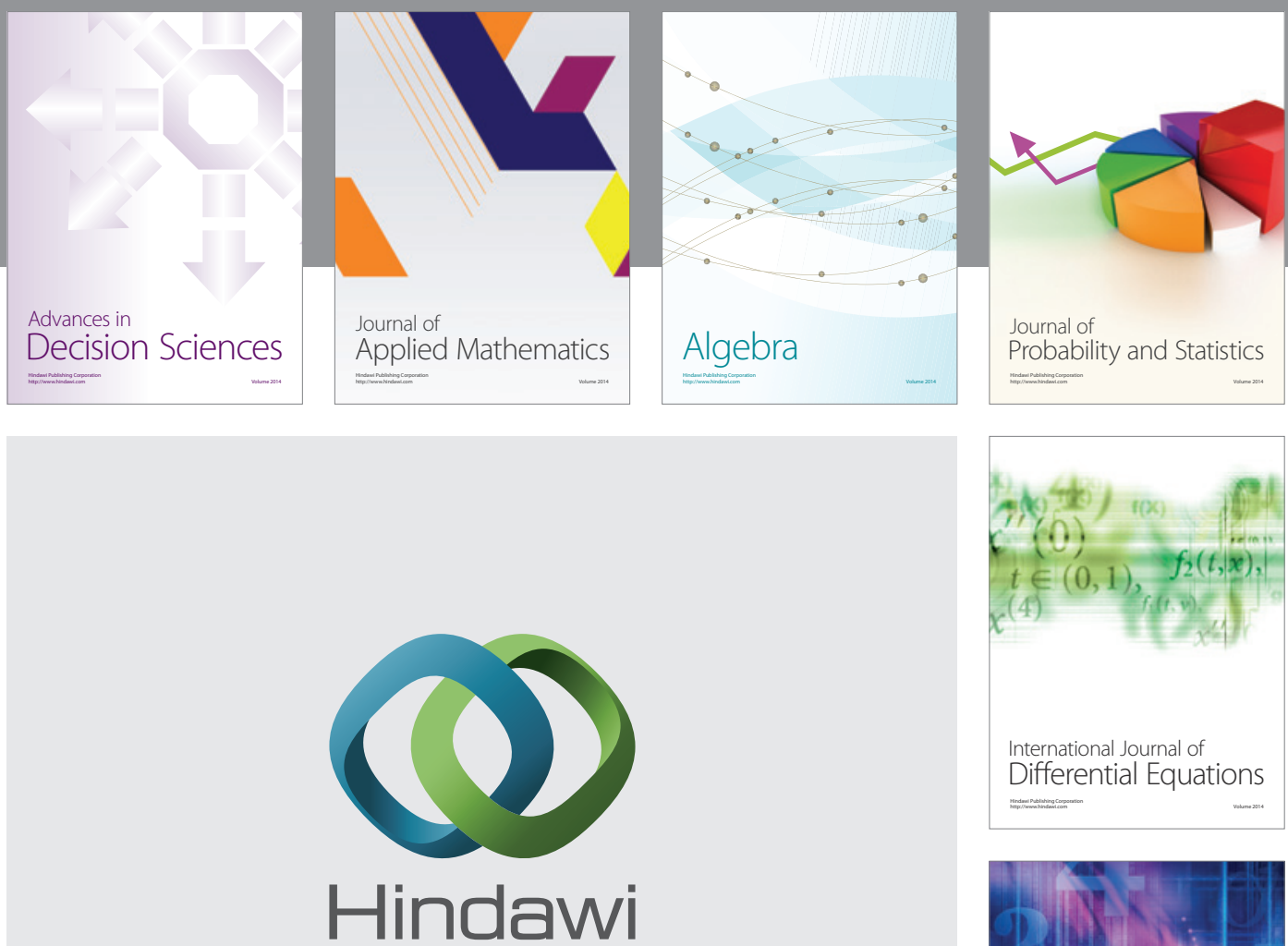

Submit your manuscripts at http://www.hindawi.com
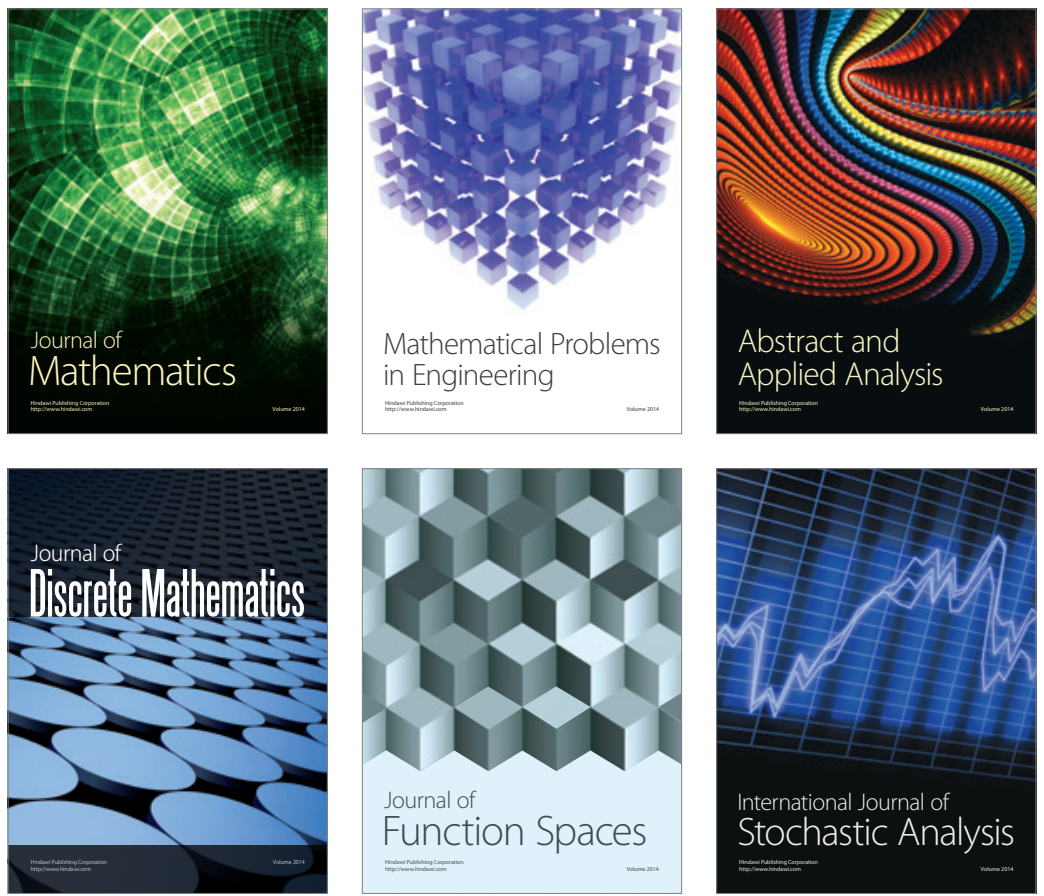

Journal of

Function Spaces

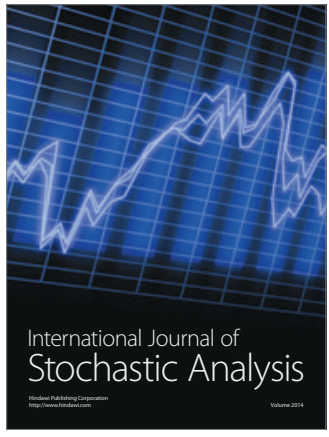

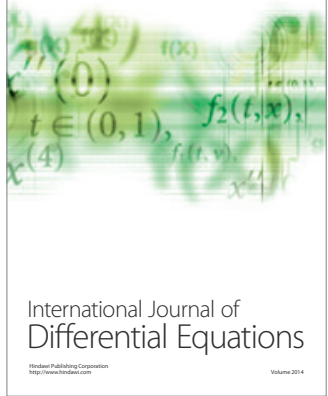
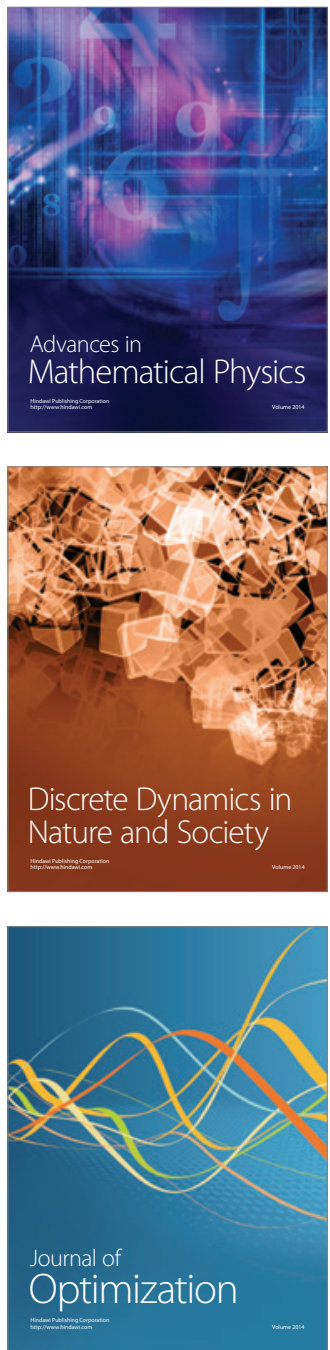